\title{
Microbiological and Histological Characteristics of Interactions Between Carvacrol and Fluconazole in a Systemic Candidiasis Animal Model
}

\author{
Zeinab Hakim¹, Fahimeh Alizadeh", Esmaeel Panahi Kokhdan², Alireza Khodavandi ${ }^{3}$ \\ 1 Department of Microbiology, Yasooj Branch, Islamic Azad University, Yasooj, Iran. \\ 2 Medicinal Plants Research Center, Yasuj University of Medical Sciences, Yasuj, Iran. \\ 3Department of Biology, Gachsaran Branch, Islamic Azad University, Gachsaran, Iran.
}

\begin{abstract}
The objectives of the study were to explore the effect of carvacrol on Candida tropicalis in vitro and in animal model, alone and in combination with fluconazole. The activity of carvacrol alone and in combination with fluconazole were tested on $C$. tropicalis using the CLSI reference method and in murine models. Carvacrol in combination with fluconazole showed satisfactory activity, with FICI mainly in the range of 0.375-1.00. Microbiologically, treatment with carvacrol alone in combination with fluconazole decreased the fungal load of kidneys of infected animal at negligible level of the number of colony counts of $C$. tropicalis. Histologically, in treated animals, no Candida organisms were found in the kidney tissues; this was in contrast to control groups in which many yeasts mixed with hyphae were observed. Carvacrol alone in combination with fluconazole as an alternative approach could be considered as the extensive opportunities for the treatment and prevention of systemic candidiasis.
\end{abstract}

Keywords: Animal models; Candida tropicalis; Candidiasis; in vitro

\section{INTRODUCTION}

The rapid emergence of non-albicans Candida species are major public health importance, notably Candida tropicalis, C. parapsilosis, C. krusei and C. glabrata. Candida species account for about $80 \%$ of fatal systemic infection,

\footnotetext{
*Corresponding Author: Fahimeh Alizadeh, e-mail: mnalizadeh@yahoo.com Zeinab Hakim ORCID Number: 0000-0002-5704-8369 Fahimeh Alizadeh ORCID Number: 0000-0002-7074-0464

Esmaeel Panahi Kokhdan ORCID Number: 0000-0002-8470-8844

Alireza Khodavandi ORCID Number: 0000-0001-9498-9822

(Received 02 October 2019, accepted 28 November 2019)
} 
where the most frequent cause of fungal infections in immunocompromised persons. The distribution of Candida species influenced by geographical location and healthcare factors ${ }^{1-6}$. In this context, $C$. tropicalis emerged as the most common non-albicans Candida species ${ }^{7}$. The reasons for dominance of C. tropicalis and its resistance to fluconazole have been difficult to clear ${ }^{8}$. Evidence suggests that marked shift in the epidemiological profile of Candida species are associated with increasing use of antifungal drugs ${ }^{1,9}$.

The currently available antifungal drugs which are used for fungal infections, have the limitation of drugs resistance and high toxicity of the compounds ${ }^{10}$. Novel therapeutic strategies are needed to counter non-albicans Candida species infections. Natural products are the most important source for promising antimicrobials, the majority of which are surprisingly found in plants ${ }^{11,12}$. To combat the continual emergence of non-albicans Candida species, there is a critical need to develop new strategies. Combination therapies are the language of antimicrobial interactions, evolved to mediate non-albicans Candida species $^{3,13,14}$. Within natural products, carvacrol (2-methyl-5-[1-methylethyl] phenol), a phenolic monoterpenoid with its pharmacological ability including antimicrobial, antioxidant, anti-inflammatory, antitumor activities, antispasmodic and antiangiogenic, have become promising therapeutic potential ${ }^{11,12,15,16}$.

Antifungal activity of carvacrol against $C$. tropicalis has also been reported ${ }^{17-20}$. However, the activity of carvacrol against $C$. tropicalis in animal model is not known. Recent reports showed that combinatorial therapy of antifungal drugs and carvacrol is very effective to eradicate Candida infections ${ }^{21,22}$. In fact, this armamentarium of therapeutic drugs provides substantial benefits in terms of rapid effect of the antifungal therapy, wide drug spectrum and potency of drug activity, synergy, lowered toxicity and reduced risk of antifungal resistance ${ }^{10,23}$.

We hypothesized that natural products in combination with antifungal drugs inhibit yeast cells growth. In this study, we explored the effect of carvacrol on $\mathrm{C}$. tropicalis in vitro and in animal model, alone and in combination with fluconazole.

\section{METHODOLOGY}

\section{Candida tropicalis}

Two clinical isolates of $C$. tropicalis $\mathrm{SN} 1$ and $\mathrm{SN2}$ and $C$. tropicalis ATCC 750 were used in this study. Clinical isolates from the vagina of patients with recurrent vulvovaginal candidiasis in Yasooj, were kindly provided by Microbiology Laboratory, Cellular and Molecular Research Center, Yasuj University of Medical Sciences (Iran). C. tropicalis cells were subcultured in Sabouraud 
Dextrose Agar (SDA; Difco Laboratories, USA) and chromogenic culture media (CHROMagar Company, France). All isolates were stored at $-80{ }^{\circ} \mathrm{C}$ in Sabouraud Dextrose Broth (SDB; Difco Laboratories, US) and supplemented with $300 \mu \mathrm{g} / \mathrm{ml}$ of chloramphenicol and isolates sterile maintained in $20 \%(\mathrm{v} / \mathrm{v})$ glycerol.

\section{In vitro Studies}

Suspensions of $C$. tropicalis $\left(5 \times 10^{2}-2.5 \times 10^{3}\right.$ cells $\left./ \mathrm{ml}\right)$ were incubated in the U-bottomed 96-well polystyrene microtiter plate at $35^{\circ} \mathrm{C}$ for 24 to $72 \mathrm{~h}$ with RPMI 1640 medium without sodium bicarbonate and with L-glutamine (Sigma-Aldrich Chemicals Co. St. Louis, MO, USA) [buffered to pH 7.0 with 0.165 M morpholinepropanesulfonic acid (MOPS, Sigma-Aldrich)] and added a range of carvacrol (Sigma-Aldrich) concentrations between 0.049 and 100 $\mu \mathrm{g} / \mathrm{ml}$ and fluconazole (Merck, Darmstadt, Germany) concentrations between 0.03125 and $64 \mu \mathrm{g} / \mathrm{ml}$ in Dimethyl sulfoxide (DMSO, Sigma-Aldrich) alone or in combination (1:1 ratio). Yeast cell- and drug-free controls were used as sterile and growth controls, respectively. The minimum inhibitory concentrations (MICs) were determined for each isolate in accordance with Clinical and Laboratory Standards Institute (CLSI) guidelines (CLSI M27-A3). Referring to the MICs of carvacrol and fluconazole alone and in combination, fractional inhibitory concentration index (FICI) was calculated for clinical isolates of $C$. tropicalis as described earlier ${ }^{24}$. The combining effect of carvacrol with fluconazole against clinical isolates of $C$. tropicalis interpreted as follows: Synergy, FICI $\leq 0.5$, partial synergy FICI $>0.5$ but $<1.0$, additive FICI=1.0, Indifference FICI $>1.0$ but $<4.0$, and antagonism $\mathrm{FICI} \geq 4.0$.

\section{In vivo Murine Studies}

Female BALB/c mice (Animal Breeding Stock Facility of Pasteur Institute of Iran, Karaj, Iran), weighing $25 \mathrm{~g}$ to $30 \mathrm{~g}$, were used according to experimental protocol approved by the Islamic Azad University Animal Ethics Committee (IR.IAU.YASOOJ.REC.1396.12), which adheres to international procedures for animal care.

The mice ( $\mathrm{n}=10$ per group) were infected with $5 \times 10^{6}$ yeast cells $/ \mathrm{ml}$ suspensions of $C$. tropicalis ATCC 750 diluted to $200 \mu$ l with sterile PBS by intravenous (i.v.) injection. At $1 \mathrm{~h}$ following the infection, mice were treated with 5 $\mathrm{mg} / \mathrm{kg} /$ day of i.v. injections of carvacrol and intraperitoneal (i.p.) injections of fluconazole alone or combination in a final volume of $200 \mu \mathrm{l} /$ mouse for 5 days. The untreated control animals received $200 \mu \mathrm{l} /$ mouse of sterile PBS.

For the systemic candidiasis study, at 2, 4, 7, 14 and 30 days after infection, 
mice were sacrificed and kidneys was collected for evaluation of microbiological characteristics following the methods described by Khodavandi et al ${ }^{25}$. Briefly, kidney tissues were homogenized in $1 \mathrm{ml}$ of sterile normal saline and serial dilutions plated on SDA to obtain the numbers of viable $C$. tropicalis ATCC 750 per gram of tissue and identified the fungal loads.

At day 14 after infection, mice were sacrificed and their kidneys were harvested and formalin fixed, dehydrated and embedded in paraffin wax using a Shandon Automated Tissue Processor (ThermoShandon, PA, USA). Sectioning of tissue samples was done at 4-5 $\mu \mathrm{m}$ and samples were stained with haematoxylin and eosin (H\&E) and periodic acid-Schiff (PAS). Final slides were then mounted and viewed under Leica microscope (DMRA II, Germany). Images were captured at $400 \times$ and $1000 \times$ magnification to visualize fungi and the intensity of the inflammatory response.

\section{Ethical Approval}

All procedures performed in studies involving human participants, which are obtained from Microbiology Laboratory, Cellular and Molecular Research Center, Yasuj University of Medical Sciences, were in accordance with the ethical standards of the institutional and/or national research committee and with the 1964 Helsinki declaration. The animal studies were approved by Islamic Azad University Animal Ethics Committee, which adheres to international procedures for animal care.

\section{Statistical Analysis}

Data were normalized with Kolmogorov-Smirnov test. Differences between means of values were compared for significance with one way ANOVA and Tukey's post-hoc test. ANOVA with repeated measure and nonparametric tests including Kruskal-Wallis test followed by Bonferroni's post hoc test were used when appropriate. Data shown reflect means \pm standard division of the means. Significant findings are denoted as: ${ }^{*} p<0.01$; and ${ }^{* *} p<0.001$ in each figure. SPSS Statistics (SPSS Inc., Chicago, IL, USA) v. 24 software was used for statistical analysis.

\section{RESULTS AND DISCUSSION}

In order to investigate the antifungal activity of carvacrol or fluconazole alone and in combination against clinical isolates of $C$. tropicalis, FICI of a combination of carvacrol and fluconazole was determined based on the MICs of carvacrol and fluconazole alone and in combination. Through antifungal activity assay, result show that carvacrol in combination with fluconazole exhibited significantly greater inhibitory activity towards clinical isolates of $C$. tropicalis 
(Table 1). Specifically, the MIC range for carvacrol alone and in combination with fluconazole which exhibited antifungal inhibition, were $4.00 \pm 0.00 \mu \mathrm{g} / \mathrm{ml}$ to $10.00 \pm 0.00 \mu \mathrm{g} / \mathrm{ml}$ and $1.00 \pm 0.00 \mu \mathrm{g} / \mathrm{ml}$ to $2.50 \pm 0.00 \mu \mathrm{g} / \mathrm{ml}$, respectively for C. tropicalis. Calculation of the FICI produced values of $0.375-1.00$, indicating significant synergism, partial synergism and indifferent. The MIC with synergistic and partial synergistic effects of carvacrol and fluconazole were markedly decreased by 4- and 8-fold in C. tropicalis ATCC 750 and C. tropicalis $\mathrm{SN} 2$, respectively compared to the MICs of carvacrol and the fluconazole alone.

Table 1. The combined antifungal effects of carvacrol with fluconazole against Candida tropicalis evaluated by FICl.

\begin{tabular}{|c|c|c|c|c|}
\hline $\begin{array}{c}\text { Antifungal } \\
\text { agents }\end{array}$ & Isolates & MICs $(\boldsymbol{\mu g} / \mathbf{m l})$ & FICI & Interpretation \\
\hline \multirow{4}{*}{ Carvacrol } & C. tropicalis ATCC 750 & $10.00 \pm 0.00$ & & \\
\cline { 2 - 5 } & C. tropicalis SN1 & $4.00 \pm 0.00$ & & \\
\cline { 2 - 5 } & C. tropicalis SN2 & $8.00 \pm 0.00$ & & \\
\hline \multirow{4}{*}{ Fluconazole } & C. tropicalis ATCC 750 & $0.50 \pm 0.00$ & & \\
\cline { 2 - 5 } & C. tropicalis SN1 & $0.25 \pm 0.00$ & & \\
\cline { 2 - 5 } & C. tropicalis SN2 & $0.125 \pm 0.00$ & & \\
\hline \multirow{2}{*}{ Carvacrol/ } & C. tropicalis ATCC 750 & $2.5 \pm 0.00 / 0.0625 \pm 0.00$ & 0.375 & Synergy \\
\cline { 2 - 5 } Fluconazole & C. tropicalis SN1 & $2.00 \pm 0.00 / 0.125 \pm 0.00$ & 1.00 & Indifference \\
\cline { 2 - 5 } & C. tropicalis SN2 & $1.00 \pm 0.00 / 0.0625 \pm 0.00$ & 0.75 & Partial synergy \\
\hline
\end{tabular}

Data are means \pm standard deviation of three independent experiments.

To determine whether these effects operated in an in vivo setting, we conducted the impact of a carvacrol or fluconazole alone and in combination in systemic candidiasis animal model. The efficacy of combination therapy was evaluated based on tissue fungal burden of kidneys and tissue histopathology. We found that mice exposure to carvacrol or fluconazole alone and in combination caused a decrease in the fungal load of kidneys $(p<0.01$ and $p<0.0001)$ (Figure 1). 


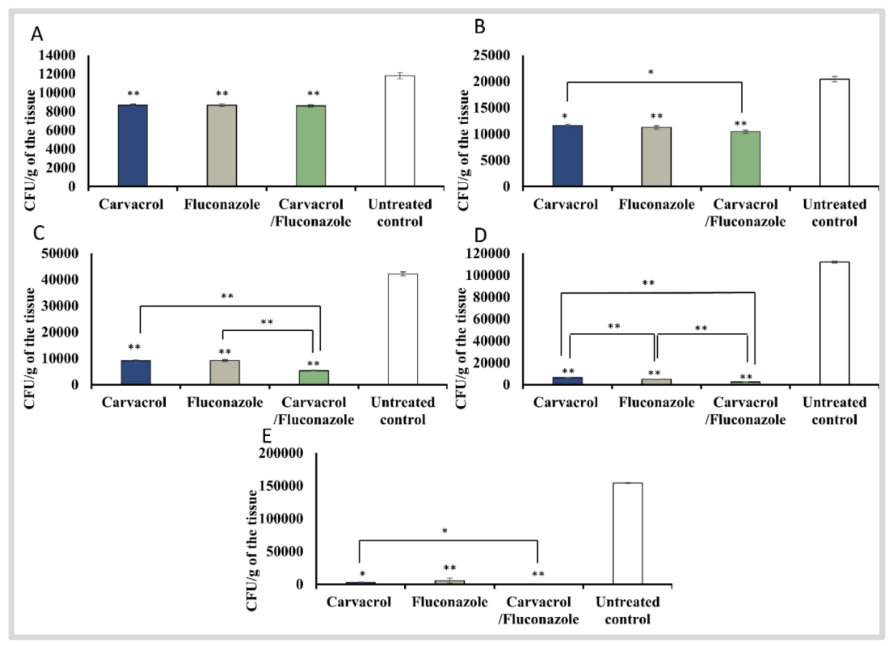

Figure 1. Fungal load of kidneys in systemically infected BALB/c mice treated or not with carvacrol or fluconazole alone and in combination, at days 2 (A), 4 (B), 7 (C), 14 (D) and 30 (E) after infection with $C$. tropicalis ATCC 750 . Data are means \pm SD of three independent ( ${ }^{*} p$ $<0.01$ and $\left.{ }^{* *} p<0.0001\right)$.

Carvacrol or fluconazole alone and in combination inhibited systemic candidiasis during the first 2 days of infection comparing to their control groups ( $p$ $<0.0001)$. There was no statistically significant difference between the two treated groups with carvacrol or fluconazole alone and in combination in the fungal load of kidneys (Tukey post hoc, $p>0.05$ ). We also noted a significant difference in Kruskal-Wallis test on the fungal load of kidneys; as expected, carvacrol or fluconazole alone and in combination exposure elicited a decrease in number of the fungal load of kidneys after 4 days of infection $(p<0.0001)$. Post hoc Bonferroni's multiple comparison test indicated a significant reduction of the fungal load of kidneys in carvacrol and combination of carvacrol and fluconazole treated groups $(p<0.01)$. While carvacrol or fluconazole alone and in combination reduced the fungal load of kidneys between treated groups after 7 days of infection $(p<0.0001)$, there was no statistically significant difference in the fungal load of kidneys between the carvacrol and fluconazole treated groups (Tukey post hoc, $p>0.05$ ). At 14 days after infection, one-way ANOVA indicated a statistically significant difference between groups ( $p<0.0001)$. Interestingly, significant difference between the two treated groups were observed in fungal load of kidneys (Tukey post hoc, $p<0.0001$ ). In the kidneys at 30 days after infection indicated a significant reduction of fungal load in treated groups comparing to their control groups. Repeated measures ANOVA indicated considerable difference between two groups of carvacrol alone and 
in combination with fluconazole at different time points $(p<0.0001)$. No significant differences were found between two groups after treatment with fluconazole alone $(p=0.058)$.

The presence of microabscesses with fungal elements was confirmed in kidney tissue in the untreated group. The most predominant forms of $C$. tropicalis in the kidney tissue were yeast mixed with hyphae (Figure 2).

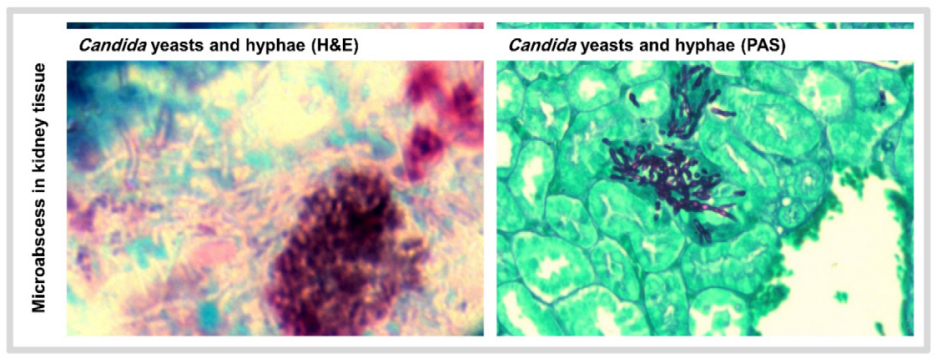

Figure 2. The presence of microabscesses with yeast mixed with hyphae of $\mathrm{C}$. tropicalis in kidney tissue from BALB/c (H\&E and PAS $\times 400)$.

The kidneys showed moderate congestion and cellular density in the untreated group. Treatments of systemically infected BALB/c mice with carvacrol and fluconazole alone and in combination presented a normal appearance of kidney tissue (Figure 3).

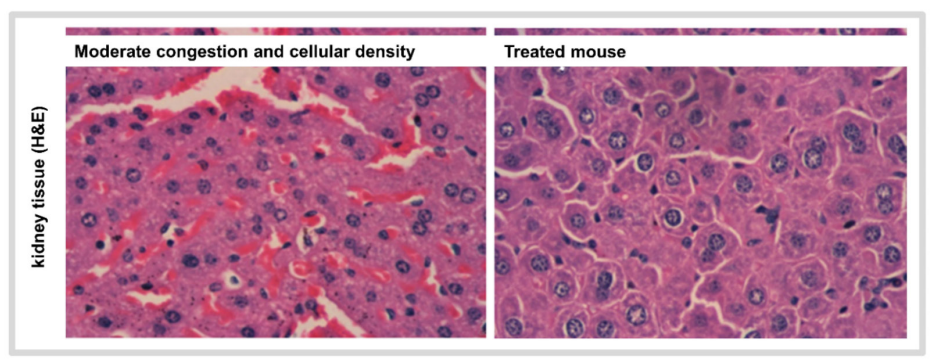

Figure 3. Moderate congestion and cellular density in systemically infected BALB/c mice and treated mouse with carvacrol and fluconazole alone and in combination (H\&E $\times 400)$.

Through our in vitro and in vivo assessment, we show carvacrol in combination with fluconazole present a promising effect in the antifungal armamentarium. Our application of carvacrol in combination with fluconazole displayed an increase of their antifungal activity against $C$. tropicalis. The unique chemical defenses of plant phenolic compounds, such as carvacrol, have been a fruitful discovery resource in the past few years ${ }^{11,26,27}$. Inhibition of $C$. tropicalis high- 
lights the value of carvacrol as a source of bioactive molecules ${ }^{17,20,28}$. Importantly, carvacrol alone and in combination with fluconazole retain high efficacy in mouse models of systemic $C$. tropicalis infection.

Several research studies have shown that the plant phenolic compounds triggered attachment of the cell surface and penetrate the phospholipid bilayer of the cell membrane afterwards. The accumulation of plant phenolic compounds disturbed structural integrity of cell membrane, and detrimentally affect the overall cell metabolism, eventually leading to cell death. $p$-Cymene is an aromatic monoterpene consists of a benzene ring without any functional groups on its side chains which is the precursor of carvacrol. $p$-Cymene is not an effective antimicrobial when used alone, but it potentiate the activity of compounds like carvacrol ${ }^{11,27,29}$. This effect has been ascribed to the accumulation of carvacrol and $p \square$ cymene in the lipid phase of the membrane and caused an expansion of the phospholipids bilayer increasing spaces through which ion leakage might occur ${ }^{30,31}$. In Escherichia coli, carvacrol and $p$-cymene impact protein synthesis and cell motility ${ }^{30}$.

Carvacrol alone and in combination with fluconazole appear a particularly valuable source of antimicrobials in vitro and in systemic candidiasis mouse model. High overall mortality and high rate of antifungal resistance to many known antifungals have made Candida infections a global health burden. Combinatorial antifungal therapy has emerged as a promising alternative approach in the treatment of Candida infections ${ }^{32}$. Fluconazole block ergosterol biosynthesis by inhibiting fungal cytochrome $\mathrm{P}_{450}$-dependent enzyme lanosterol 14- $\alpha$-demethylase ${ }^{33334}$. Many mechanisms involved in the synergistic activity of antifungal agents. The inhibition of different stages in the fungal intracellular pathways that are essential for cell survival, increase uptake of an antifungal agent resulting from the action of another antifungal agent on the fungal cell wall or cell membrane and reach their target fungal DNA, the inhibition of carrier proteins and the simultaneous inhibition of different cell targets ${ }^{14,35}$. Studies demonstrated that carvacrol combined with fluconazole suggested as an approach to achieve synergy and expand fluconazole spectrum ${ }^{21,22}$. The synergistic combination of carvacrol with fluconazole might be explained by the carvacrol promoting the effects of fluconazole, mainly on the cell wall, cell membrane and other membrane structures of yeast. Additionally, carvacrol damage the fungal cell membrane may facilitate the entrance of fluconazole to the cell, thereby leading to a higher effect on inhibition of ergosterol biosynthesis. Moreover, this issue might induce apoptosis in yeast cell by reactive oxygen species accumulation ${ }^{33: 36,37}$. This raises the possibility that carvacrol may also turn the fungistatic action of fluconazole into a fungicidal action ${ }^{33: 36}$. 
Many murine models of systemic candidiasis have been reported ${ }^{25,38,39}$. Carvacrol alone and in combination with fluconazole inhibited $C$. tropicalis growth in a murine systemic candidiasis model. Microbiological and histopathological changes suggest that carvacrol alone and in combination with fluconazole can prevent candidiasis by inhibiting growth and morphogenesis of $C$. tropicalis. Carvacrol was previously reported to inhibit hyphal growth ${ }^{40-43}$. The results of our present study were in agreement with the previous report that carvacrol significantly reduced the number of CFU sampled from the oral and vaginal candidiasis in immunosuppressed rats. In addition, no hyphal colonization of the epithelium and lumina of the vagina were seen in animals treated with carvacrol ${ }^{40,44,45}$. Similar results were obtained from Manohar et al ${ }^{42}$ experiments.

Antifungals developed from combinatorial therapy are the foundation of modern medicine and have saved millions of lives. The promise of carvacrol in combination with fluconazole as a source of antifungals has the potential to reinvigorate the stagnated antifungal discovery pipelines. Our validation of carvacrol in combination with fluconazole as an alternative approach demonstrates the extensive opportunities for the treatment and prevention of systemic candidiasis after further investigations and sufficient clinical trials.

\section{ACKNOWLEDGEMENTS}

The authors acknowledge Dr. Sadegh Nouripour-Sisakht from the Cellular and Molecular Research Center, Yasuj University of Medical Sciences, Yasuj, Iran for kindly providing the clinical isolates of $C$. tropicalis. The authors wish to thank the Islamic Azad University of Yasooj for financial support. The results presented in this study is part of the Master thesis of Zeinab Hakim. 


\section{REFERENCES}

1. Juyal, D.; Sharma, M.; Pal, S.; Rathaur, V.K.; Sharma, N. Emergence of non-albicans Candida species in neonatal candidemia. N. Am. J. Med. Sci. 2013, 5, 541-245.

2. Kołaczkowska, A.; Kołaczkowski, M. Drug resistance mechanisms and their regulation in non-albicans Candida species. J. Antimicrob. Chemother. 2016, 71, 1438-1450.

3. Makanjuola, O.; Bongomin, F.; Fayemiwo, S.A. An update on the roles of non-albicans Candida species in vulvovaginitis. J. Fungi (Basel). 2018, 4, E121.

4. Mushi, M.F.; Mtemisika, C.; Bader, O.; Bii, C.; Mirambo, M.M.; Groß, U.; Mshana, S.E. High oral carriage of non-albicans Candida spp. among HIV-infected individuals. Int. J. Infect. Dis. 2016, 49, 185-188.

5. Whaley, S.G.; Berkow, E.L.; Rybak, J.M.; Nishimoto, A.T.; Barker, K.S.; Rogers, P.D. Azole antifungal resistance in Candida albicans and emerging non-albicans Candida species. Front Microbiol. 2017, 7, 2173.

6. Whibley, N.; Gaffen, S.L. Beyond Candida albicans: mechanisms of immunity to non-albicans Candida species. Cytokine. 2015, 76, 42-52.

7. Zuza-Alves, D.L.; Silva-Rocha, W.P.; Chaves, G.M. An update on Candida tropicalis based on basic and clinical approaches. Front Microbiol. 2017, 8, 1927.

8. Kothavade, R.J.; Kura, M.M.; Valand, A.G.; Panthaki, M.H. Candida tropicalis: its prevalence, pathogenicity and increasing resistance to fluconazole. J. Med. Microbiol. 2010, 59, 873-880.

9. Bongomin, F.; Gago, S.; Oladele, R.O.; Denning, D.W. Global and multi-national prevalence of fungal diseases-estimate precision. J. Fungi (Basel). 2017, 3, E57.

10. Scorzoni, L.; de Paula E Silva, A.C.; Marcos, C.M.; Assato, P.A.; de Melo, W.C.; de Oliveira, H.C.; Costa-Orlandi, C.B.; Mendes-Giannini, M.J.; Fusco-Almeida, A.M. Antifungal therapy: new advances in the understanding and treatment of mycosis. Front Microbiol. 2017, 8, 36.

11. Chouhan, S.; Sharma, K.; Guleria, S. Antimicrobial activity of some essential oils-present status and future perspectives. Medicines (Basel). 2017, 4, E58.

12. Rajput, J.D.; Bagul, S.D.; Pete, U.D.; Zade, C.M.; Padhye, S.B.; Bendre, R.S. Perspectives on medicinal properties of natural phenolic monoterpenoids and their hybrids. Mol. Divers.

2018, 22, 225-245.

13. Budzyńska, A.; Różalska, S.; Sadowska, B.; Różalska, B. Candida albicans/Staphylococcus aureus dual-species biofilm as a target for the combination of essential oils and fluconazole or mupirocin. Mycopathologia. 2017, 182, 989-995.

14. Campitelli, M.; Zeineddine, N.; Samaha, G.; Maslak, S. Combination antifungal therapy: a review of current data. J. Clin. Med. Res. 2017, 9, 451-456.

15. Beltrán, J.M.; Esteban, M.A. Properties and applications of plants of origanum sp. genus. SM. J. Biol. 2016, 2, 1006.

16. Cacciatore, I.; Di Giulio, M.; Fornasari, E.; Di Stefano, A.; Cerasa, L.S.; Marinelli, L.; Turkez, H.; Di Campli, E.; Di Bartolomeo, S.; Robuffo, I.; Cellini, L. Carvacrol codrugs: a new approach in the antimicrobial plan. PLoS One. 2015, 10, e0120937.

17. Alizadeh, F.; Khodavandi, A.; Esfandyari, S.; Nouripour-Sisakht, S. Analysis of ergosterol and gene expression profiles of sterol $\Delta 5,6$-desaturase $\left(E R G_{3}\right)$ and lanosterol $14 \alpha$-demethylase (ERG11) in Candida albicans treated with carvacrol. J. Herbmed Pharmacol. 2018, 7, 79-87. 
18. Gallucci, M.N.; Carezzano, M.E.; Oliva, M.M.; Demo, M.S.; Pizzolitto, R.P.; Zunino, M.P.; Zygadlo, J.A.; Dambolena, J.S. In vitro activity of natural phenolic compounds against fluconazole-resistant Candida species: a quantitative structure-activity relationship analysis. $J$. Appl. Microbiol. 2014, 116, 795-804.

19. Piras, A.; Cocco, V.; Falconieri, D.; Porcedda, S.; Marongiu, B.; Maxia, A.; Frau, M.A.; Gonçalves, M.J.; Cavaleiro, C.; Salgueiro, L. Isolation of the volatile oil from Satureja thymbra by supercritical carbon dioxide extraction: chemical composition and biological activity. Nat. Prod. Commun. 2011, 6, 1523-1526.

20. Soliman, S.; Alnajdy, D.; El-Keblawy, A.A.; Mosa, K.A.; Khoder, G.; Noreddin, A.M. Plants' natural products as alternative promising anti-Candida drugs. Pharmacogn. Rev. 2017, 11, 104-122.

21. Doke, S.K.; Raut. J.S.; Dhawale, S.; Karuppayil, S.M. Sensitization of Candida albicans biofilms to fluconazole by terpenoids of plant origin. J. Gen. Appl. Microbiol. 2014, 6o, 163-168.

22. Khodavandi, A.; Alizadeh, F.; Alizadeh, E. Antifungal activity of carvacrol in combination with fluconazole or amphotericin B against Candida albicans. Malaysian J. Microbiol. 2018, 14, 356-363.

23. Chang, Y.L.; Yu, S.J.; Heitman, J.; Wellington, M.; Chen, Y.L. New facets of antifungal therapy. Virulence. 2016, 8, 222-236.

24. Khodavandi, A.; Alizadeh, F.; Aala, F.; Sekawi, Z.; Chong, P.P. In vitro investigation of antifungal activity of allicin alone and in combination with azoles against Candida species. Mycopathologia. 2010, 169, 287-295.

25. Khodavandi, A.; Alizadeh, F.; Harmal, N.; Sidik, S.M.; Othman, F.; Sekawi, Z.; Jahromi, M.A.; Ng, K.P.; Chong, P.P. Comparison between efficacy of allicin and fluconazole against Candida albicans in vitro and in a systemic candidiasis mouse model. FEMS Microbiol. Lett. 2011, 315, 87-93.

26. Naghdi Badi, H.; Abdollahi, M.; Mehrafarin, A.; Ghorbanpour, M.; Tolyat, M.; Qaderi, A.; Ghiaci Yekta, M. An overview on two valuable natural and bioactive compounds, thymol and carvacrol, in medicinal plants. J. Med. Plants. 2017, 3, 1-32.

27. Zhang, Y.; Muend, S.; Rao, R. Dysregulation of ion homeostasis by antifungal agents. Front. Microbiol. 2012, 3, 133 .

28. Sharifi-Rad, M.; Varoni, E.M.; Iriti, M.; Martorell, M.; Setzer, W.N.; Del Mar Contreras, M.; Salehi, B.; Soltani-Nejad, A.; Rajabi, S.; Tajbakhsh, M.; Sharifi-Rad, J. Carvacrol and human health: a comprehensive review. Phytother. Res. 2018, 32, 1675-1687.

29. Lv, F.; Liang, H.; Yuan, Q.; Li, C. In vitro antimicrobial effects and mechanism of action of selected plant essential oil combinations against four food related microorganisms. Food Res. Int. 2011, 44, 3057-3064.

30. Marchese, A.; Arciola, C.R.; Barbieri, R.; Silva, A.S.; Nabavi, S.F.; Tsetegho Sokeng, A.J.; Izadi, M.; Jafari, N.J.; Suntar, I.; Daglia, M.; Nabavi, S.M. Update on monoterpenes as antimicrobial agents: a particular focus on p-cymene. Materials (Basel). 2017, 10, E947.

31. Morsy, N.F.S. Chemical structure, quality indices and bioactivity of essential oil constituents. Active Ingredients from Aromatic and Medicinal Plants. HA El-Shemy. 2017.

32. Spitzer, M.; Robbins, N.; Wright, G.D. Combinatorial strategies for combating invasive fungal infections. Virulence. 2017, 8, 169-185.

33. Khan, M.S.; Malik, A.; Ahmad, I. Anti-candidal activity of essential oils alone and in combination with amphotericin B or fluconazole against multi-drug resistant isolates of Candida 
albicans. Med. Mycol. 2012, 5o, 33-42.

34. Vazquez, J.A. Combination antifungal therapy against Candida species: the new frontier-are we there yet? Med. Mycol. 2003, 41, 355-368.

35. Johnson, M.D.; MacDougall, C.; Ostrosky-Zeichner, L.; Perfect, J.R.; Rex, J.H. Combination antifungal therapy. Antimicrob. Agents Chemother. 2004, 48, 693-715.

36. Scalas, D.; Mandras, N.; Roana, J.; Tardugno, R.; Cuffini, A.M.; Ghisetti, V.; Benvenuti, S.; Tullio, V. Use of Pinus sylvestris L. (Pinaceae), Origanum vulgare L. (Lamiaceae), and Thymus vulgaris L. (Lamiaceae) essential oils and their main components to enhance itraconazole activity against azole susceptible/not-susceptible Cryptococcus neoformans strains. BMC Complement Altern. Med. 2018, 18, 143.

37. Sharma, M.; Manoharlal, R.; Negi, A.S.; Prasad, R. Synergistic anticandidal activity of pure polyphenol curcumin I in combination with azoles and polyenes generates reactive oxygen species leading to apoptosis. FEMS Yeast Res. 2010, 10, 570-578.

38. Diba, A.; Alizadeh, F. In vitro and in vivo antifungal activity of Allium hirtifolium and Allium sativum. Avicenna J. Phytomed. 2018, 8, 465-474.

39. Segal, E.; Frenkel, M. Experimental in vivo models of candidiasis. J. Fungi (Basel). 2018, 4, E21.

40. Chami, F.; Chami, N.; Bennis, S.; Trouillas, J.; Remmal, A. Evaluation of carvacrol and eugenol as prophylaxis and treatment of vaginal candidiasis in an immunosuppressed rat model. J. Antimicrob. Chemother. 2004, 54, 909-914.

41. Khodavandi, A.; Alizadeh, F.; Zaboli zadeh, S.H. Inhibitory effect of carvacrol on the expression of Candida albicans hyphae-specific gene (HWP1). JBUMS. 2018, 2O, 63-70.

42. Manohar, V.; Ingram, C.; Gray, J.; Talpur, N.A.; Echard, B.W.; Bagchi, D.; Preuss, H.G. Antifungal activities of origanum oil against Candida albicans. Mol. Cell Biochem. 2001, 228, 111-117.

43. Ocak, I.; Çelik, A.; Özel, M.Z.; Korcan, E.; Konuk, M. Antifungal activity and chemical composition of essential oil of Origanum hypericifolium. Int. J. Food Properties. 2012, 15, $38-48$.

44. Chami, N.; Chami, F.; Bennis, S.; Trouillas, J.; Remmal, A. Antifungal treatment with carvacrol and eugenol of oral candidiasis in immunosuppressed rats. Braz. J. Infect. Dis. 2oo4, 8, 217-226.

45. Chami, N.; Bennis, S.; Chami, F.; Aboussekhra, A.; Remmal, A. Study of anticandidal activity of carvacrol and eugenol in vitro and in vivo. Oral Microbiol. Immunol. 2005, 20, 106-111. 